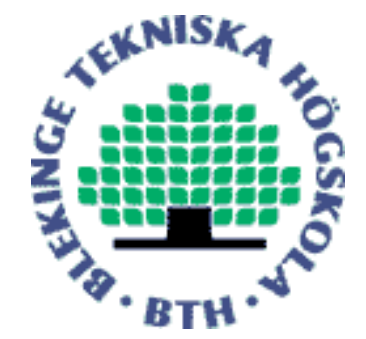

Copyright (C) 2014 IEEE.

Citation for the published paper:

On Physical Layer Security for Reactive DF Cognitive Relay Networks

Louis Sibomana, Hans-Jürgen Zepernick, Hung Tran

IEEE Global Communications Conference

2014 Austin, USA

This material is posted here with permission of the IEEE. Such permission of the IEEE does not in any way imply IEEE endorsement of any of BTH's products or services Internal or personal use of this material is permitted. However, permission to reprint/republish this material for advertising or promotional purposes or for creating new collective works for resale or redistribution must be obtained from the IEEE by sending a blank email message to pubs-permissions@iee.org.

By choosing to view this document, you agree to all provisions of the copyright laws protecting it. 


\title{
On Physical Layer Security for Reactive DF Cognitive Relay Networks
}

\author{
Louis Sibomana $^{1}$, Hans-Jürgen Zepernick ${ }^{1}$, and Hung Tran ${ }^{2}$ \\ ${ }^{1}$ Blekinge Institute of Technology, SE-371 79 Karlskrona, Sweden \\ E-mail: $\{1 \mathrm{sm}, \mathrm{hjz}\} @$ bth.se \\ ${ }^{2}$ Information Technology Faculty, NIEM, 31-Phan Dinh Giot, Hanoi, Vietnam \\ E-mail: $\{$ tranhungemail\}@gmail.com
}

\begin{abstract}
This paper analyzes the physical layer security for cognitive relay networks under the peak interference power constraint of the primary user receiver. In particular, a secondary user (SU) transmitter communicates with an SU receiver through the help of multiple secondary relays (SRs) using a decodeand-forward (DF) protocol. There exist multiple eavesdroppers (EAVs) who illegally listen to the secondary network communication. We consider a reactive DF scheme, and only the SRs that satisfy a decoding threshold participate in the relay selection. Analytical expressions of the probability of existence of secrecy capacity and secrecy outage probability are obtained. Numerical results are provided to evaluate the impact of the number of SRs, number of EAVs and channel mean powers on the secondary system security. We also investigate the effect of the interference from the primary network to the secondary network performance. Moreover, the performance of proactive DF is analyzed for the purpose of comparison.
\end{abstract}

\section{INTRODUCTION}

Recently, cooperative relaying has been introduced in cognitive radio networks (CRNs) to enhance the secondary network coverage and to improve radio spectrum utilization [1]-[4]. In particular, secondary relays (SRs) assist the secondary user transmitter (SU-Tx) to forward the message to the SU receiver (SU-Rx) using the licensed frequency band of the primary user (PU). In this context, the secondary system capacity [5] and outage probability [6] have been analyzed for reactive decode and forward (R-DF) and proactive DF (P-DF) schemes considering either peak interference power (PIP) or outage constraints at the PU receiver (PU-Rx).

In addition, information theoretic security has received much attention [7]-[10] where physical layer properties such as multiple antennas and cooperative networks are exploited to achieve secure transmission in wireless communications. In [10]-[13], different relay selection strategies have been studied for conventional cooperative wireless networks in the presence of eavesdroppers (EAVs) that intercept the legitime message transmission. Moreover, CRN technology is also prone to eavesdropping attacks as discussed in [14]. Cognitive relay physical layer security has been considered in [15] where the SU asymptotic secrecy rate and secrecy outage probability have been analyzed. However, the work in [15] ignored the effect of the PU transmitter (PU-Tx) interference to the secondary network and thus, the analysis does not depict a realistic scenario. Further, the analysis in [10], [12], [13],
[15] assumed high signal-to-noise ratio (SNR) and hence, all relays are able to successfully decode the message from the source. This assumption is not valid for practical systems that often operate in the low to medium SNR regimes. It is noted that the secondary network operates in low to medium SNR to satisfy the PU interference power constraint. Therefore, it is important to evaluate the secondary system security at all SNRs and by considering the interference from the PU-Tx to the secondary network.

In this paper, we study the physical layer security for a secondary relay network with multiple SRs in the presence of multiple EAVs and consider the interference from the primary network to the secondary network. We consider an R-DF scheme, i.e., only the SRs that satisfy the decoding threshold participate in the relay selection. In particular, we derive the cumulative distribution function (CDF) and probability density function (PDF) of the end-to-end signal-to-interference-plusnoise ratio (SINR). Accordingly, analytical expressions of the probability of existence of non-zero secrecy capacity and secrecy outage probability are obtained. Moreover, we investigate the impact of the number of SRs, number of EAVs and interference from the PU-Tx to SU-Rx and EAV on the secondary network security. For the purpose of comparison, the performance of proactive DF is also analyzed.

The rest of the paper is organized as follows. Section II describes the system model. In Section III, we present the performance analysis of the considered system. Section IV provides numerical results and discussions. Finally, conclusions are presented in Section V.

\section{SYSTEM MODEL}

\section{A. Underlay cognitive relay network model}

Fig.1 shows an underlay cognitive relay network where an SU-Tx sends confidential messages to the SU-Rx through the assistance of $N$ trusted SRs in the presence of a PUTx communicating with a PU-Rx. There exist $K$ passive EAVs intercepting signals from the SR to the SU-Rx. We assume that the direct links from the SU-Tx to the SURx and EAV are not available due to severe shadowing [12], [13]. The secondary network communication occurs in two phases. In the first phase, the SU-Tx broadcasts the information to all SRs. Then, one SR is selected to forward 


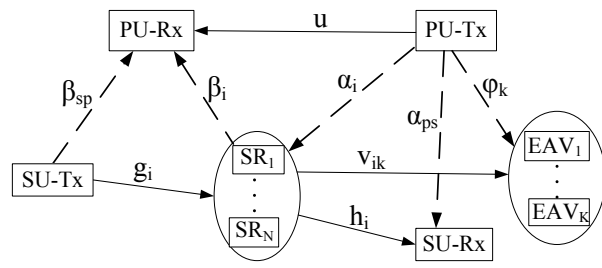

Fig. 1. Model of the considered cognitive relay network in the presence of multiple eavesdroppers. (Dashed lines: Interference links; Solid lines: Communication links).

the message to the SU-Rx in the second phase based on R$\mathrm{DF}$ or P-DF protocols. The channel power gains of the links $\mathrm{PU}-\mathrm{Tx} \rightarrow \mathrm{PU}-\mathrm{Rx}, \mathrm{SU}-\mathrm{Tx} \rightarrow \mathrm{SR}_{i}, \mathrm{SR}_{i} \rightarrow \mathrm{SU}-\mathrm{Rx}$ and $\mathrm{SR}_{i} \rightarrow \mathrm{EAV}_{k}$ are, respectively, denoted by $u, g_{i}, h_{i}, i \in\{1,2, \ldots, N\}$ and $v_{i k}, k \in\{1,2, \ldots, K\}$. Further, $\beta_{s p}, \beta_{i}, \alpha_{i}, \alpha_{p s}$ and $\varphi_{k}$ represent the channel power gains of the $\mathrm{SU}-\mathrm{Tx} \rightarrow \mathrm{PU}$ $\mathrm{Rx}, \mathrm{SR}_{i} \rightarrow \mathrm{PU}-\mathrm{Rx}, \mathrm{PU}-\mathrm{Tx} \rightarrow \mathrm{SR}_{i}, \mathrm{PU}-\mathrm{Tx} \rightarrow \mathrm{SU}-\mathrm{Rx}$ and PU$\mathrm{Tx} \rightarrow \mathrm{EAV}_{k}$ links, respectively. We assume that all channels are subject to independent Rayleigh fading and the channel power gains are exponentially distributed random variables (RVs). Accordingly, the channel mean powers are defined as $\Omega_{u}, \Omega_{g_{i}}, \Omega_{h_{i}}, \Omega_{v_{i k}}, \Omega_{s p}, \Omega_{\beta_{i}}, \Omega_{\alpha_{i}}, \Omega_{p s}$ and $\Omega_{\varphi_{k}}$.

For the underlay approach, the interference from the SU transmitting nodes $\left(\mathrm{SU}-\mathrm{Tx}, \mathrm{SR}_{i}\right.$ ) to the PU-Rx should be managed to be below a predefined threshold [2], [3] such that

$$
\begin{gathered}
P_{s} \beta_{s p} \leq Q \\
P_{r_{i}} \beta_{i} \leq Q
\end{gathered}
$$

where $Q$ is the maximum acceptable level of interference that the PU-Rx can tolerate. Further, $P_{s}$ and $P_{r_{i}}$ are, respectively, the SU-Tx and $i$ th SR instantaneous transmit powers. Note that the transmit power cannot be infinite or very large in practice. As such, $P_{s} \leq P_{s, \max }$ and $P_{r_{i}} \leq P_{r, \max }$ where $P_{s, \max }$ and $P_{r, \text { max }}$ denote the SU-Tx and $i$ th SR maximum transmit power limits, respectively. We can see from (1) that perfect channel state information (CSI) of the PU is required at the SU-Tx to maintain the PU-Rx PIP constraint, i.e., perfect knowledge of $\beta_{s p}$ at the SU-Tx. In practice, it is hard for the SU to obtain perfect CSI of the PU and is costly in terms of feedback. To overcome the limitation of PU perfect CSI, we can tolerate a certain amount of error for the SU transmit power control [4]. Accordingly, the interference power constraint at the PU-Rx is reformulated as

$$
\operatorname{Pr}\left\{P_{s} \beta_{s p}>Q\right\} \leq \epsilon
$$

where $\epsilon$ is the tolerated error to satisfy (1). As a consequence, the SU-Tx and $i$ th SR transmit powers are obtained as

$$
\begin{gathered}
P_{s}=\min \left\{\frac{Q}{\Omega_{s p} \ln (1 / \epsilon)}, P_{s, \max }\right\} \\
P_{r_{i}}=\min \left\{\frac{Q}{\Omega_{\beta_{i}} \ln (1 / \epsilon)}, P_{r, \max }\right\}
\end{gathered}
$$

Note that this case happens when statistical information about the PU channel is available at the SUs, i.e., $\Omega_{s p}$ and $\Omega_{\beta_{i}}$ are relatively stable and can be estimated at the SU transmitting nodes [3].

\section{B. $R-D F$ scheme and secrecy capacity}

We define $C_{s, i}, C_{i, d}$ and $C_{i, k}$ as the instantaneous channel capacity at the $\mathrm{SR}_{i}, \mathrm{SU}-\mathrm{Rx}$ and $\mathrm{EAV}_{k}$ nodes, respectively. Taking account for the PU-Tx interference, we have

$$
\begin{aligned}
C_{s, i} & =\frac{W}{2} \log _{2}\left(1+\frac{\gamma_{s} g_{i}}{\gamma_{p} \alpha_{i}+1}\right) \\
C_{i, d} & =\frac{W}{2} \log _{2}\left(1+\frac{\gamma_{r_{i}} h_{i}}{\gamma_{p} \alpha_{p s}+1}\right) \\
C_{i, k} & =\frac{W}{2} \log _{2}\left(1+\frac{\gamma_{r_{i}} v_{i k}}{\gamma_{p} \varphi_{k}+1}\right)
\end{aligned}
$$

where $W$ is the system bandwidth. Further, $\gamma_{s}=P_{s} / N_{0}$, $\gamma_{r_{i}}=P_{r_{i}} / N_{0}$ and $\gamma_{p}=P_{p} / N_{0}$ are, respectively, the SU-Tx, $\mathrm{SR}_{i}$ and PU-Tx transmit SNRs with $N_{0}$ being the noise power. Moreover, $\gamma_{s, \text { max }}=P_{s, \max } / N_{0}$ and $\gamma_{r, \max }=P_{r, \max } / N_{0}$ denote the maximum transmit SNR of the SU-Tx and $\mathrm{SR}_{i}$, respectively. We also define $\gamma_{Q}=Q / N_{0}$ as the PU PIP SNR.

For the DF protocol, each SR first decodes the received signal from the SU-Tx and then re-encodes and transmits its decoded outcome to the SU-Rx. In the opportunistic R-DF scheme, the best SR among the set of SR candidates that successfully decoded the message from the SU-Tx [5], [6] is selected to forward the message to the SU-Rx as

$$
\mathbf{b}=\arg \max _{i \in \mathcal{R}_{j}}\left\{\gamma_{i, d}\right\}, \quad \mathcal{R}_{j}=\left\{j \mid \gamma_{s, i} \geq \gamma_{t h}, \forall i \in \mathcal{N}\right\}
$$

where $\mathcal{N}=\{1,2, \ldots, N\}, \gamma_{i, d}=\left(\gamma_{r_{i}} h_{i}\right) /\left(\gamma_{p} \alpha_{p s}+1\right)$ and $\gamma_{s, i}=\left(\gamma_{s} g_{i}\right) /\left(\gamma_{p} \alpha_{i}+1\right)$. Further, $\gamma_{t h}=2^{\frac{2 R_{s}}{W}}-1$ is the decoding threshold with $R_{s}$ being the $\mathrm{SU}-\mathrm{Tx} \rightarrow \mathrm{SR}_{i}$ link target transmission rate and $\mathcal{R}_{j}$ is a set of $j$ SRs that decoded the message. Accordingly, the SINR at the SU-Rx is given by

$$
\gamma_{\mathrm{R}-\mathrm{DF}}=\max _{i \in \mathcal{R}_{j}}\left\{\gamma_{i, d}\right\}
$$

During the second phase, $K$ EAVs independently overhear the transmission from $\mathrm{SR}_{i}$ to the SU-Rx. The secrecy rate at the SU-Rx can be achieved by considering the EAV with the highest SINR as

$$
\gamma_{\mathbf{b}, E}=\max _{k \in \mathcal{K}}\left\{\frac{\gamma_{r} v_{\mathbf{b}, k}}{\gamma_{p} \varphi_{k}+1}\right\}, \quad \mathcal{K}=\{1,2, \ldots, K\}
$$

Given (10) and (11), and in view of (7) and (8), we have $C_{\mathrm{R}-\mathrm{DF}}=(W / 2) \log _{2}\left(1+\gamma_{\mathrm{R}-\mathrm{DF}}\right)$ and $C_{\mathbf{b}, E}=(W / 2) \log _{2}(1+$ $\left.\gamma_{\mathbf{b}, E}\right)$. The secrecy capacity is defined as the difference between the capacity of the main link and that of a wiretap link [8]. The SU achievable secrecy capacity is expressed as

$$
C_{\mathrm{sec}}=\max \left\{0, C_{\mathrm{R}-\mathrm{DF}}-C_{\mathbf{b}, E}\right\}
$$

For further analysis, we define the RV $Y=a X_{1} /\left(b X_{2}+c\right)$ where $X_{1}$ and $X_{2}$ are independent exponentially distributed RVs with mean $\Omega_{1}$ and $\Omega_{2}$, respectively. Further, $a, b$ and $c$ are positive constants. The CDF of $Y$ is given by [6]

$$
F_{Y}(y)=1-\frac{a \Omega_{1}}{a \Omega_{1}+b \Omega_{2} y} \exp \left(-\frac{c y}{a \Omega_{1}}\right)
$$


By differentiating (13) with respect to $y$, the PDF of $Y$ is

$$
f_{Y}(y)=\frac{a b \Omega_{1} \Omega_{2} \exp \left(-\frac{c y}{a \Omega_{1}}\right)}{\left(a \Omega_{1}+b \Omega_{2} y\right)^{2}}+\frac{a c \Omega_{1} \exp \left(-\frac{c y}{a \Omega_{1}}\right)}{a \Omega_{1}+b \Omega_{2} y}
$$

\section{PERformance ANALYSis}

In this section, analytical expressions of the probability of non-zero secrecy capacity and secrecy outage probability are derived. We first consider the presence of a single EAV and then, extend the results to multiple EAVs. In addition, we assume that all SRs are close to each other to form a cluster [4] and thus, $\Omega_{g_{i}}=\Omega_{g}, \Omega_{h_{i}}=\Omega_{h}, \Omega_{\beta_{i}}=\Omega_{\beta}, \Omega_{\alpha_{i}}=\Omega_{\alpha}$ and $\gamma_{r_{i}}=\gamma_{r}, \forall i \in \mathcal{N}$. The same assumption is also applied to the $K$ EAVs and hence, $\Omega_{\varphi_{k}}=\Omega_{\varphi}$ and $\Omega_{v_{\mathbf{b}, k}}=\Omega_{v}, \forall k \in \mathcal{K}$.

\section{A. Presence of a Single EAV}

Let $F_{\gamma_{\mathrm{R}-\mathrm{DF}}}(y)$ and $f_{\gamma_{\mathbf{b}, E}}(y)$ denote, respectively, the CDF of $\gamma_{\mathrm{R}-\mathrm{DF}}$ and PDF of $\gamma_{\mathbf{b}, E}$. From (10), we have

$$
F_{\gamma_{\mathrm{R}-\mathrm{DF}}}(y)=\sum_{j=0}^{N} \underbrace{\operatorname{Pr}\left\{\gamma_{\mathrm{R}-\mathrm{DF}}<y \mid \mathcal{R}_{j}\right\}}_{P_{1}} \operatorname{Pr}\left\{\mathcal{R}_{j}\right\}
$$

where $\operatorname{Pr}\left\{\mathcal{R}_{j}\right\}$ represents the probability of exact $j$ SRs satisfying the decoding threshold and is obtained as

$$
\operatorname{Pr}\left\{\mathcal{R}_{j}\right\}=\left(\begin{array}{c}
N \\
j
\end{array}\right) A^{N-j}(1-A)^{j}
$$

In (16), $A$ is determined with the help of (13) as

$$
A=\operatorname{Pr}\left\{\frac{\gamma_{s} g_{i}}{\gamma_{p} \alpha_{i}+1} \leq \gamma_{t h}\right\}=1-\frac{\exp \left(-B_{0} \gamma_{t h}\right)}{1+B \gamma_{t h}}
$$

where $B_{0}=1 /\left(\gamma_{s} \Omega_{g}\right)$ and $B=B_{0} \gamma_{p} \Omega_{\alpha}$. We consider that the events $\mathcal{R}_{j}$ and $\gamma_{\mathrm{R}-\mathrm{DF}}<y$ are independent [6] and therefore,

$$
\begin{aligned}
P_{1} & =\int_{0}^{\infty} \operatorname{Pr}\left\{\max _{i \in \mathcal{R}_{j}}\left\{\frac{\gamma_{r} h_{i}}{\gamma_{p} x+1}\right\}<y\right\} f_{\alpha_{p s}}(x) d x \\
& =\int_{0}^{\infty}\left\{1-\exp \left[-\frac{y\left(\gamma_{p} x+1\right)}{\gamma_{r} \Omega_{h}}\right]\right\}^{j} \frac{\exp \left(-\frac{x}{\Omega_{p s}}\right)}{\Omega_{p s}} d x \\
& =\sum_{m=0}^{j}\left(\begin{array}{c}
j \\
m
\end{array}\right)(-1)^{m} \frac{\exp \left(-B_{2} y\right)}{1+B_{1} y}
\end{aligned}
$$

where $B_{1}=B_{2} \gamma_{p} \Omega_{p s}$ and $B_{2}=m /\left(\gamma_{r} \Omega_{h}\right)$. Substituting (18) and (16) into (15), we obtain

$$
\begin{aligned}
F_{\gamma_{\mathrm{R}-\mathrm{DF}}}(y) & =\sum_{j=0}^{N} \sum_{n=0}^{N-j} \sum_{m=0}^{j}\left(\begin{array}{c}
N \\
j
\end{array}\right)\left(\begin{array}{c}
N-j \\
n
\end{array}\right)\left(\begin{array}{c}
j \\
m
\end{array}\right)(-1)^{m+n} \\
& \times \frac{\exp \left\{-\left[(n+j) B_{0} \gamma_{t h}+B_{2} y\right]\right\}}{\left(1+B \gamma_{t h}\right)^{n+j}\left(1+B_{1} y\right)}
\end{aligned}
$$

Further, the PDF of $\gamma_{\mathbf{b}, E}$ is obtained using (14) as

$$
f_{\gamma_{\mathbf{b}, E}}(y)=\frac{G \exp (-G y)}{\left(1+G_{1} y\right)}+\frac{G_{1} \exp (-G y)}{\left(1+G_{1} y\right)^{2}}
$$

where $G=1 /\left(\gamma_{r} \Omega_{v}\right)$ and $G_{1}=G \gamma_{p} \Omega_{\varphi}$.
1) Existence of non-zero secrecy capacity: According to [8], [13], the probability of existence of a non-zero secrecy capacity denoted by $P_{\mathrm{R}-\mathrm{DF}}^{\mathrm{ex}}$ is given by

$$
P_{\mathrm{R}-\mathrm{DF}}^{\mathrm{ex}}=\operatorname{Pr}\left\{C_{\mathrm{sec}}>0\right\}=1-\int_{0}^{\infty} F_{\gamma_{\mathrm{R}-\mathrm{DF}}}(y) f_{\gamma_{\mathbf{b}, E}}(y) d y
$$

Then, substituting (19) and (20) into (21), we have

$$
\begin{aligned}
P_{\mathrm{R}-\mathrm{DF}}^{\mathrm{ex}} & =1-\sum_{j=0}^{N} \sum_{n=0}^{N-j} \sum_{m=0}^{j}\left(\begin{array}{c}
N \\
j
\end{array}\right)\left(\begin{array}{c}
N-j \\
n
\end{array}\right)\left(\begin{array}{c}
j \\
m
\end{array}\right)(-1)^{m+n} \\
& \times \frac{\exp \left[-(n+j) B_{0} \gamma_{t h}\right]}{\left(1+B \gamma_{t h}\right)^{n+j}} I_{1}
\end{aligned}
$$

where $\lambda=B_{2}+G$ and

$$
I_{1}=\int_{0}^{\infty} \frac{\exp (-\lambda y)}{1+B_{1} y}\left[\frac{G}{1+G_{1} y}+\frac{G_{1}}{\left(1+G_{1} y\right)^{2}}\right] d y
$$

To solve (23), two cases are considered: If $B_{1} \neq G_{1}, I_{1}$ is obtained in (24) where $\Psi(z)=\exp (z) \operatorname{Ei}(-z)$ with $\operatorname{Ei}(z)=$ $-\int_{-z}^{\infty} \frac{\exp (-t)}{t} d t$ being the exponential integral function. If $B_{1}=G_{1}, I_{1}$ is given by

$$
I_{1}=\frac{G}{B_{1}}+\frac{1}{2}-\frac{\lambda}{2 B_{1}}+\frac{\lambda}{B_{1}^{2}}\left(G-\frac{\lambda}{2}\right) \Psi\left(\frac{\lambda}{B_{1}}\right)
$$

Note that (24) and (25) are solved with the help of [16, eq. (3.352.4)], [16, eq. (3.353.3)] and [16, eq. (3.353.2)].

2) Secrecy outage probability: For delay-constrained applications and without perfect CSI of the EAV channel, the secondary network must set a secrecy target rate $R$. The secrecy outage probability is formulated as

$$
P_{\text {sec, R-DF }}^{\text {out }}=\sum_{j=0}^{N} \underbrace{\operatorname{Pr}\left\{C_{\text {sec }}<R \mid \mathcal{R}_{j}\right\}}_{P_{2}} \operatorname{Pr}\left\{\mathcal{R}_{j}\right\}
$$

The secondary system is in outage whenever the transmitted message is neither secure nor reliable [13]. By recalling that $\gamma_{\mathrm{R}-\mathrm{DF}}$ and $\mathcal{R}_{j}$ are independent and using the total probability theorem, $P_{2}$ is given by

$$
\begin{aligned}
P_{2} & =\underbrace{\operatorname{Pr}\left\{C_{\mathrm{sec}}<R \mid \gamma_{\mathrm{R}-\mathrm{DF}}>\gamma_{\mathbf{b}, E}\right\}}_{P_{3}} \operatorname{Pr}\left\{\gamma_{\mathrm{R}-\mathrm{DF}}>\gamma_{\mathbf{b}, E}\right\} \\
& +\underbrace{\operatorname{Pr}\left\{C_{\mathrm{sec}}<R \mid \gamma_{\mathrm{R}-\mathrm{DF}} \leq \gamma_{\mathbf{b}, E}\right\}}_{P_{4}} \underbrace{\operatorname{Pr}\left\{\gamma_{\mathrm{R}-\mathrm{DF}} \leq \gamma_{\mathbf{b}, E}\right\}}_{P_{5}}
\end{aligned}
$$

where $P_{5}=\int_{0}^{\infty} F_{\gamma_{\mathrm{R}-\mathrm{DF}}}(y) f_{\gamma_{\mathbf{b}, E}}(y) d y$ and $P_{3}$ is obtained as

$$
\begin{aligned}
P_{3} & =\operatorname{Pr}\left\{\gamma_{\mathrm{R}-\mathrm{DF}}<\rho\left(1+\gamma_{\mathbf{b}, E}\right)-1 \mid \gamma_{\mathrm{R}-\mathrm{DF}}>\gamma_{\mathbf{b}, E}\right\} \\
& =\int_{0}^{\infty} \frac{F_{\gamma_{\mathrm{R}-\mathrm{DF}}}[\rho(1+y)-1]-F_{\gamma_{\mathrm{R}-\mathrm{DF}}}(y)}{\operatorname{Pr}\left\{\gamma_{\mathrm{R}-\mathrm{DF}}>\gamma_{\mathbf{b}, E}\right\}} f_{\gamma_{\mathbf{b}, E}}(y) d y
\end{aligned}
$$

where $\rho=2^{\frac{2 R}{W}}$. Since $R>0$, we have $P_{4}=1$. Substituting (28) into (27), we obtain

$$
P_{2}=\int_{0}^{\infty} F_{\gamma_{\mathrm{R}-\mathrm{DF}}}[\rho(1+y)-1] f_{\gamma_{\mathbf{b}, E}}(y) d y
$$




$$
\begin{aligned}
I_{1} & =\frac{G B_{1}}{B_{1}-G_{1}} \int_{0}^{\infty} \frac{\exp (-\lambda y)}{1+B_{1} y} d y-\frac{G G_{1}}{B_{1}-G_{1}} \int_{0}^{\infty} \frac{\exp (-\lambda y)}{1+G_{1} y} d y+\frac{G_{1} B_{1}^{2}}{\left(G_{1}-B_{1}\right)^{2}} \int_{0}^{\infty} \frac{\exp (-\lambda y)}{1+B_{1} y} d y-\frac{B_{1} G_{1}^{2}}{\left(G_{1}-B_{1}\right)^{2}} \\
& \times \int_{0}^{\infty} \frac{\exp (-\lambda y)}{1+G_{1} y} d y+\frac{G_{1}^{2}}{G_{1}-B_{1}} \int_{0}^{\infty} \frac{\exp (-\lambda y)}{\left(1+G_{1} y\right)^{2}} d y \\
& =\frac{G}{B_{1}-G_{1}}\left[\Psi\left(\frac{\lambda}{G_{1}}\right)-\Psi\left(\frac{\lambda}{B_{1}}\right)\right]+\frac{\lambda}{G_{1}-B_{1}} \Psi\left(\frac{\lambda}{G_{1}}\right)+\frac{G_{1}}{G_{1}-B_{1}}+\frac{B_{1} G_{1}}{\left(G_{1}-B_{1}\right)^{2}}\left[\Psi\left(\frac{\lambda}{G_{1}}\right)-\Psi\left(\frac{\lambda}{B_{1}}\right)\right]
\end{aligned}
$$

where $F_{\gamma_{\mathrm{R}-\mathrm{DF}}}[\rho(1+y)-1]$ can be obtained from (18). Further, (26) is obtained by considering (16), (20) and (29) as

$$
\begin{aligned}
P_{\mathrm{R}-\mathrm{DF}}^{\text {out }} & =\sum_{j=0}^{N} \sum_{n=0}^{N-j} \sum_{m=0}^{j}\left(\begin{array}{c}
N \\
j
\end{array}\right)\left(\begin{array}{c}
N-j \\
n
\end{array}\right)\left(\begin{array}{c}
j \\
m
\end{array}\right)(-1)^{m+n} \\
& \times \frac{\exp \left\{-\left[(n+j) B_{0} \gamma_{t h}+B_{2}(\rho-1)\right]\right\}}{\left[1+B_{1}(\rho-1)\right]\left(1+B \gamma_{t h}\right)^{n+j}} I_{2}
\end{aligned}
$$

where $A_{3}=\left(B_{1} \rho\right) /\left[1+B_{1}(\rho-1)\right], \delta=B_{2} \rho+G$ and

$$
\begin{aligned}
I_{2} & =\frac{1}{2}+\frac{G}{G_{1}}-\frac{\delta}{2 G_{1}}+\left[\frac{G \delta}{G_{1}^{2}}-\frac{\delta^{2}}{2 G_{1}^{2}}\right] \Psi\left(\frac{\delta}{G_{1}}\right) \\
I_{2} & =\frac{G}{A_{3}-G_{1}}\left[\Psi\left(\frac{\delta}{G_{1}}\right)-\Psi\left(\frac{\delta}{A_{3}}\right)\right]+\frac{\delta}{G_{1}-A_{3}} \Psi\left(\frac{\delta}{G_{1}}\right) \\
& +\frac{G_{1}}{G_{1}-A_{3}}+\left[\Psi\left(\frac{\delta}{G_{1}}\right)-\Psi\left(\frac{\delta}{A_{3}}\right)\right] \frac{A_{3} G_{1}}{\left(G_{1}-A_{3}\right)^{2}}
\end{aligned}
$$

It is noted that (31) for $A_{3}=G_{1}$ and (32) for $A_{3} \neq G_{1}$ are obtained using the same integration as in (24) and (25).

\section{B. Presence of multiple EAVs}

1) Existence of non-zero secrecy capacity: In the presence of multiple EAVs, the PDF of $\gamma_{\mathbf{b}, E}$ is obtained from (11) and by using (14) as

$$
\begin{aligned}
f_{\gamma_{\mathbf{b}, E}}(y) & =K \sum_{l=0}^{K-1}\left(\begin{array}{c}
K-1 \\
l
\end{array}\right)(-1)^{l} \exp [-(l+1) G y] \\
& \times\left[\frac{G}{\left(1+G_{1} y\right)^{l+1}}+\frac{G_{1}}{\left(1+G_{1} y\right)^{l+2}}\right]
\end{aligned}
$$

Then, $P_{\mathrm{R}-\mathrm{DF}}^{\mathrm{ex}}$ is obtained considering (19) and (33) as

$$
\begin{aligned}
P_{\mathrm{R}-\mathrm{DF}}^{\mathrm{ex}} & =1-K \sum_{j=0}^{N} \sum_{n=0}^{N-j} \sum_{m=0}^{j} \sum_{l=0}^{K-1}\left(\begin{array}{c}
N \\
j
\end{array}\right)\left(\begin{array}{c}
N-j \\
n
\end{array}\right)\left(\begin{array}{c}
j \\
m
\end{array}\right) \\
& \times \frac{\left(\begin{array}{c}
K-1 \\
l
\end{array}\right)(-1)^{m+l+n}}{\left(1+B \gamma_{t h}\right)^{n+j}} \exp \left[-(n+j) B_{0} \gamma_{t h}\right] I_{3}
\end{aligned}
$$

where $\mu=B_{2}+(l+1) G$ and

$$
\begin{aligned}
I_{3} & =\frac{A_{1}}{(l+1) !}\left[\sum_{t=1}^{l+1}(t-1) !\left(\frac{1}{B_{1}}\right)^{-t}(-\mu)^{l+1-t}\right. \\
& \left.-(-\mu)^{l+1} \Psi\left(\frac{\mu}{B_{1}}\right)\right]+\frac{A_{2}}{(l+2) !}\left[\sum_{t=1}^{l+2}(t-1) !\right. \\
& \left.\times\left(\frac{1}{B_{1}}\right)^{-t}(-\mu)^{l+2-t}-(-\mu)^{l+2} \Psi\left(\frac{\mu}{B_{1}}\right)\right]
\end{aligned}
$$

Further, $A_{1}=G /\left(B_{1}\right)^{l+2}$ and $A_{2}=1 /\left(B_{1}\right)^{l+2}$. Note that (35) is obtained for a particular case where $B_{1}=G_{1}$ and using [16, eq. (3.353.2)]. If $B_{1} \neq G_{1}, I_{3}$ is given by

$$
I_{3}=\int_{0}^{\infty} \frac{\exp (-\mu y)}{1+B_{1} y}\left[\frac{G}{\left(1+G_{1} y\right)^{q_{1}}}+\frac{G_{1}}{\left(1+G_{1} y\right)^{q_{2}}}\right] d y
$$

where $q_{1}=l+1$ and $q_{2}=l+2$. It is difficult to obtain a closedform solution for (36) and hence, it is solved numerically.

2) Secrecy outage probability: For multiple EAVs and by substituting (29) and (33) into (26), $P_{\mathrm{R}-\mathrm{DF}}^{\text {out }}$ is given in (37) where $A_{0}=(n+j) B_{0} \gamma_{t h}, \xi=B_{2} \rho+(l+1) G, G_{2}=$ $G /\left(G_{1}\right)^{l+2}$ and $G_{3}=1 /\left(G_{1}\right)^{l+2}$. If $A_{3} \neq G_{1}$, we have

$$
I_{4}=\int_{0}^{\infty} \frac{\exp (-\xi y)}{1+A_{3} y}\left[\frac{G}{\left(1+G_{1} y\right)^{q_{1}}}+\frac{G_{1}}{\left(1+G_{1} y\right)^{q_{2}}}\right] d y
$$

3) P-DF scheme: When the P-DF scheme is used, the best $\mathrm{SR}$ is selected to maximize the minimum of the SINR between the source-relay and relay-destination [5], [6] as

$$
\gamma_{\mathrm{P}-\mathrm{DF}}=\max _{i \in\{1,2, \ldots, N\}}\left\{\min \left\{\frac{\gamma_{s} g_{i}}{\gamma_{p} \alpha_{i}+1}, \frac{\gamma_{r_{i}} h_{i}}{\gamma_{p} \alpha_{p s}+1}\right\}\right\}
$$

As such, the CDF of $\gamma_{\mathrm{P}-\mathrm{DF}}$, i.e., $\operatorname{Pr}\left\{\gamma_{\gamma_{\mathrm{P} \text {-DF }}}<y\right\}$ is obtained as

$$
\begin{aligned}
F_{\gamma_{\mathrm{p}-\mathrm{DF}}}(y) & =\int_{0}^{\infty} \frac{\exp \left(-\frac{x}{\Omega_{p s}}\right)}{\Omega_{p s}} \prod_{i=1}^{N}\left[1-\frac{\exp \left(-B_{0} y\right)}{1+B y}\right. \\
& \left.\times \exp \left[-D\left(\gamma_{p} x+1\right) y\right]\right] d x \\
& =\sum_{j=0}^{N}\left(\begin{array}{c}
N \\
j
\end{array}\right)(-1)^{j} \frac{\exp (-j \eta y)}{(1+B y)^{j}\left(1+D_{1} y\right)}
\end{aligned}
$$

where $\eta=B_{0}+D, D=1 / \gamma_{r} \Omega_{h}$ and $D_{1}=D j \gamma_{p} \Omega_{p s}$. For the case of multiple EAVs, $P_{\mathrm{P}-\mathrm{DF}}^{\mathrm{ex}}$ is given in (42) and is solved numerically.

\section{NumericAl RESULTS}

This section provides numerical examples based on MonteCarlo simulations to verify our analysis conducted in the previous section. In all simulations, we set the system bandwidth $W=5 \mathrm{MHz}$, PU-Rx tolerable error $\epsilon=0.01$ and target transmission rate of the SU-Tx $\rightarrow$ SR link $R_{s}=64 \mathrm{kbps}$.

Fig. 2 shows that the probability of non-zero secrecy capacity generally increases with respect to the increase of PU PIP SNR $\gamma_{Q}$. This is due to the fact that increasing 


$$
\begin{aligned}
P_{\mathrm{R}-\mathrm{DF}}^{\text {out }} & =\sum_{j=0}^{N} \sum_{n=0}^{N-j} \sum_{m=0}^{j} \sum_{l=0}^{K-1}\left(\begin{array}{c}
N \\
j
\end{array}\right)\left(\begin{array}{c}
N-j \\
n
\end{array}\right)\left(\begin{array}{c}
j \\
m
\end{array}\right)\left(\begin{array}{c}
K-1 \\
l
\end{array}\right)(-1)^{m+l+n} \frac{K \exp \left\{-\left[A_{0}+B_{2}(\rho-1)\right]\right\}}{\left[1+B_{1}(\rho-1)\right]\left(1+B \gamma_{t h}\right)^{n+j}} I_{4} \\
I_{4} & =\frac{G_{2}}{(l+1) !}\left[\sum_{t=1}^{l+1}(t-1) !\left(\frac{1}{G_{1}}\right)^{-t}(-\xi)^{l+1-t}-(-\xi)^{l+1} \Psi\left(\frac{\xi}{G_{1}}\right)\right]+\frac{G_{3}}{(l+2) !}\left[\sum_{t=1}^{l+2}(t-1) !\left(\frac{1}{G_{1}}\right)^{-t}\right. \\
& \left.\times(-\xi)^{l+2-t}-(-\xi)^{l+2} \Psi\left(\frac{\xi}{G_{1}}\right)\right], \quad A_{3}=G_{1}
\end{aligned}
$$

$$
P_{\mathrm{P}-\mathrm{DF}}^{\mathrm{ex}}=1-K \sum_{j=0}^{N} \sum_{l=0}^{K-1}\left(\begin{array}{c}
N \\
j
\end{array}\right)\left(\begin{array}{c}
K-1 \\
l
\end{array}\right)(-1)^{j+l} \int_{0}^{\infty} \frac{\exp \{-[j \eta+(l+1) G] y\}}{(1+B y)^{j}\left(1+D_{1} y\right)}\left[\frac{G}{\left(1+G_{1} y\right)^{l+1}}+\frac{G_{1}}{\left(1+G_{1} y\right)^{l+2}}\right] d y
$$

$\gamma_{Q}$ allows an increase of the SU transmit SNR according to (4) and (5). Moreover, it is shown that this probability increases when the number $N$ of SRs increases. For example, by increasing $N=1$ to $N=4$, the secondary network security is improved. This shows how exploiting opportunistic relay selection in cooperative networks contributes to security enhancement, i.e., increasing the possibility that more SRs participate in relay selection. On the other hand, the secondary network security performance is degraded when the number $K$ of EAVs increases, e.g., from $K=1$ to $K=2$. In this case, the probability of intercepting the message increases. Further, the probability of existence of secrecy capacity is also reduced by increasing the PU-Tx transmit SNR $\gamma_{p}$, e.g., $\gamma_{p}=5 \mathrm{~dB}$ to $\gamma_{p}=10 \mathrm{~dB}$. Here, the PU-Tx transmit power becomes a stronger interference source to the secondary receiving nodes.

It is observed in Fig. 3 that the probability of non-zero secrecy capacity decreases as the number $K$ of EAVs becomes high for all scenarios. In addition, we can see that the secondary network security is enhanced by increasing the channel mean power $\Omega_{\varphi}$ of the PU-Tx $\rightarrow$ EAV link, i.e., $\Omega_{\varphi}=0.5$ (Case 1) to $\Omega_{\varphi}=2$ (Case 2). Here, the PU-Tx is close to the EAV, and thus, the EAV channel capacity is degraded by the PU-Tx interference. As expected, when the EAV is far away from the SR, the probability of non-zero secrecy capacity is high, e.g., $\Omega_{v}=1$ (Case 3) compared to $\Omega_{v}=2$ (Case 2).

Fig. 4 shows the secrecy outage probability. As discussed for the probability of non-zero secrecy capacity in Figs. 2 and 3 , we can see that the number $K$ of EAVs and channel mean power $\Omega_{\varphi}$ of the PU-Tx $\rightarrow$ EAV link have the same impact on the secrecy outage probability. For example, increasing $\Omega_{\varphi}=2$ to $\Omega_{\varphi}=4$, the secondary system security is improved. The effect of the SU secrecy target rate is also observed in Fig. 4 where the secrecy outage probability is high for $R=128 \mathrm{kbps}$ compared to $R=64 \mathrm{kbps}$.

Fig. 5 compares the probability of non-zero secrecy capacity for the P-DF and R-DF schemes. In general, the R-DF scheme outperforms the P-DF scheme as the PU PIP SNR $\gamma_{Q}$ increases. This is due to the fact that when $\gamma_{Q}$ increases, the decoding threshold is satisfied in the first phase. Accordingly, more relay selection diversity is achieved. However, at low

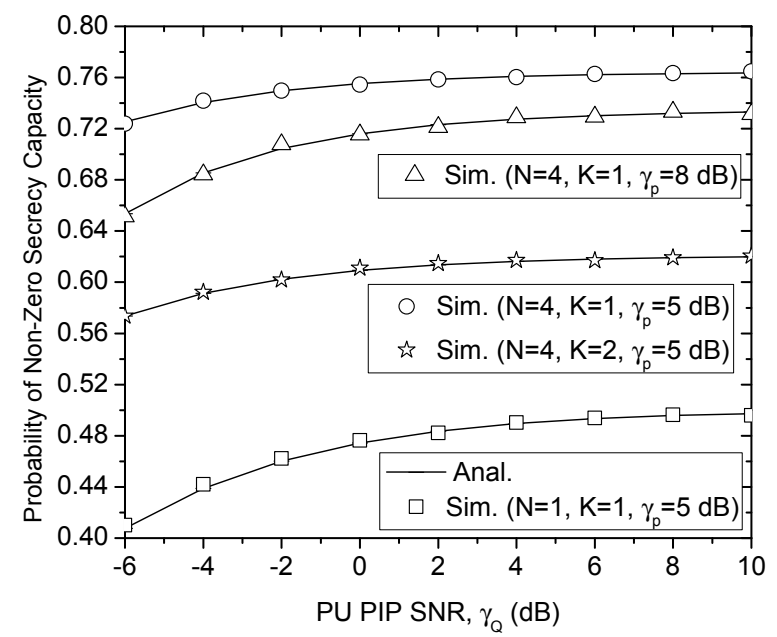

Fig. 2. Probability of existence of non-zero secrecy capacity versus PU PIP SNR, $\gamma_{Q}$ with SU maximum transmit SNR $\gamma_{s, \max }=\gamma_{r, \max }=12 \mathrm{~dB}$, $\Omega_{g}=\Omega_{h}=\Omega_{v}=2$ and $\Omega_{\alpha}=\Omega_{\beta}=\Omega_{\varphi}=\Omega_{s p}=\Omega_{p s}=0.5$.

values of $\gamma_{Q}$, e.g., at $\gamma_{Q}<-2 \mathrm{~dB}$ in Case 4 , P-DF provides a better performance than R-DF. Again, the probability of nonzero secrecy capacity is high when the interference from PU$\mathrm{Tx} \rightarrow \mathrm{EAV}$ increases, e.g., $\Omega_{\varphi}=2$ (Case 5 ) to $\Omega_{\varphi}=4$ (Case 6). In addition, the secondary network security is degraded when $K$ increases, e.g., $K=1$ in Case 5 to $K=2$ in Case 6 .

\section{CONCLUSION}

In this paper, we have studied the physical layer security for cognitive relay networks with multiple SRs in the presence of a single EAV and multiple EAVs. Analytical expressions of the probability of existence of non-zero secrecy capacity and secrecy outage probability have been derived under the PU PIP constraint and SU maximum transmit power limit. The effect of the number $N$ of SRs, number $K$ of EAVs and channel mean powers on the secondary security performance has been evaluated. Further, the impact of the interference from primary network to the secondary network has been investigated. The numerical examples indicate that the secondary system security is improved by exploiting relay selection diversity. 


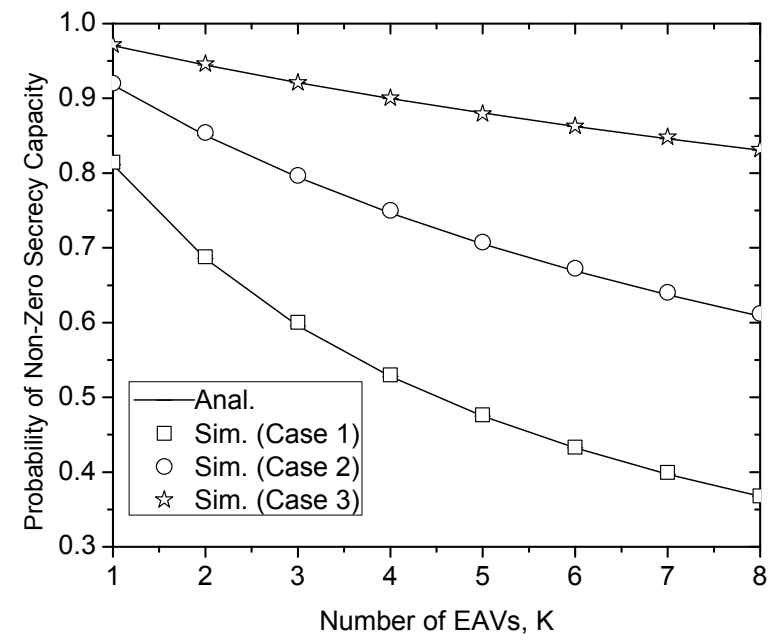

Fig. 3. Probability of existence of non-zero secrecy capacity versus number $K$ of EAVs with $N=6, \gamma_{Q}=10 \mathrm{~dB}, \gamma_{p}=5 \mathrm{~dB}, \gamma_{s, \max }=\gamma_{r, \max }=12 \mathrm{~dB}$ and $\Omega_{g}=\Omega_{h}=2$.

Case 1: $\Omega_{v}=2, \Omega_{\alpha}=\Omega_{\beta}=\Omega_{\varphi}=\Omega_{s p}=\Omega_{p s}=0.5$;

Case 2: $\Omega_{v}=2, \Omega_{\alpha}=\Omega_{\beta}=\Omega_{s p}=\Omega_{p s}=0.5, \Omega_{\varphi}=2$;

Case 3: $\Omega_{v}=1, \Omega_{\alpha}=\Omega_{\beta}=\Omega_{s p}=\Omega_{p s}=0.5, \Omega_{\varphi}=2$.

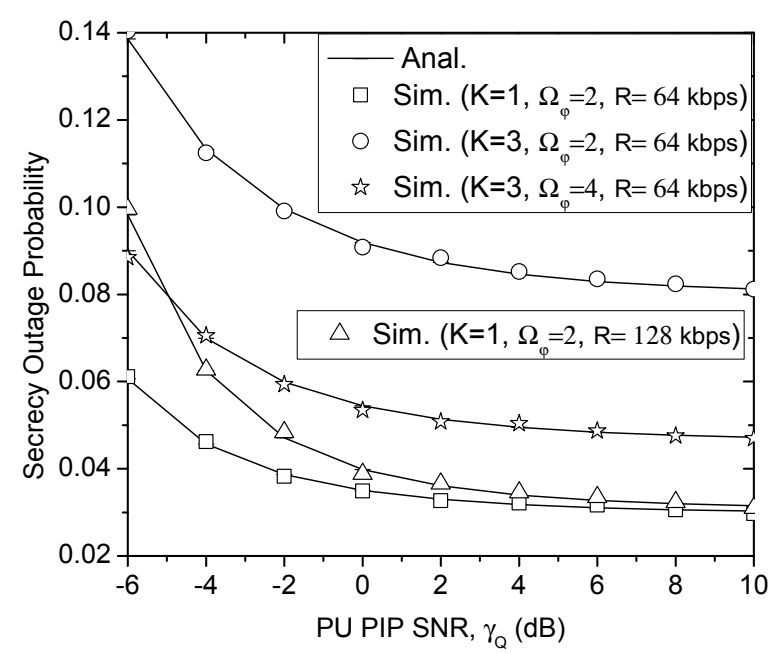

Fig. 4. Secrecy outage probability with $N=6, \gamma_{p}=5 \mathrm{~dB}, \gamma_{s, \max }=$ $\gamma_{r, \max }=12 \mathrm{~dB}, \Omega_{g}=\Omega_{h}=2, \Omega_{v}=1$ and $\Omega_{\alpha}=\Omega_{\beta}=\Omega_{s p}=\Omega_{p s}=0.5$.

The results also show that the secondary network security is enhanced by increasing the channel mean power of the PU$\mathrm{TX} \rightarrow$ EAV link.

\section{REFERENCES}

[1] Z. Qian, J. Juncheng, and Z. Jin, "Cooperative relay to improve diversity in cognitive radio networks," IEEE Commun. Mag., vol. 47, no. 2, pp. 111-117, Feb. 2009.

[2] J. Lee, H. Wang, J. G. Andrews, and D. Hong, "Outage probability of cognitive relay networks with interference constraints," IEEE Trans. Wireless Commun., vol. 10, no. 2, pp. 390-395, Feb. 2011.

[3] Y. Zou, J. Zhu, B. Zheng, and Y.-D. Yao, "An adaptive cooperation diversity scheme with best relay selection in cognitive radio networks,' IEEE Trans. Signal Process., vol. 58, no. 10, pp. 5438-5445, Oct. 2010.

[4] K. Tourki, K. A. Qaraqe, and M.-S. Alouinin, "Outage analysis for underlay cognitive networks using incremental regenerative relaying," IEEE Trans. Veh. Technol., vol. 62, no. 2, pp. 721-734, Feb. 2013.

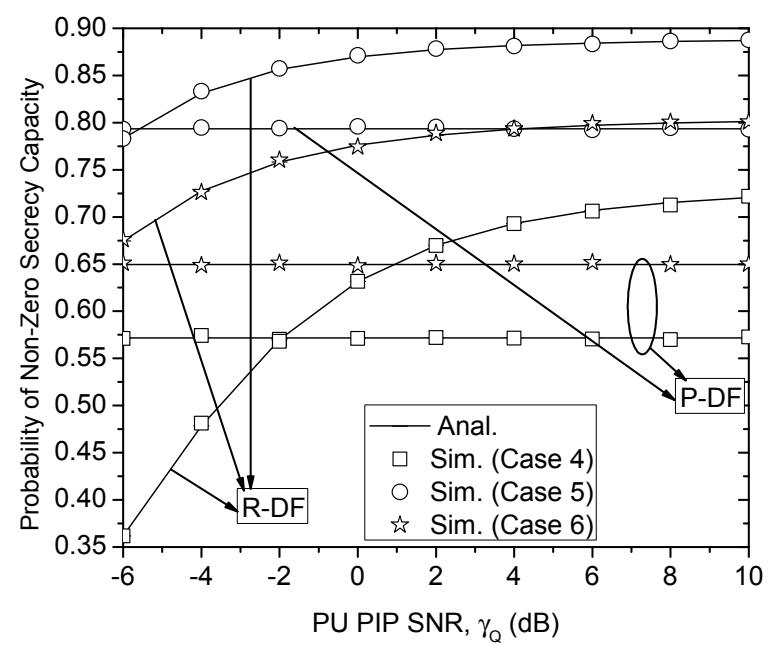

Fig. 5. Probability of existence of non-zero secrecy capacity with $N=4$, $\gamma_{s, \text { max }}=\gamma_{r, \max }=12 \mathrm{~dB}, \gamma_{p}=5 \mathrm{~dB}$ and $\Omega_{g}=\Omega_{h}=\Omega_{v}=2$.

Case 4: $K=1, \Omega_{\alpha}=\Omega_{\beta}=\Omega_{s p}=\Omega_{p s}=\Omega_{\varphi}=2$;

Case 5: $K=1, \Omega_{\alpha}=\Omega_{\beta}=\Omega_{s p}=\Omega_{p s}=1, \Omega_{\varphi}=4$

Case 6: $K=2, \Omega_{\alpha}=\Omega_{\beta}=\Omega_{s p}=\Omega_{p s}=1, \Omega_{\varphi}=4$

[5] S. Sagong, J. Lee, and D. Hong, "Capacity of reactive DF scheme in cognitive relay networks," IEEE Trans. Wireless Commun., vol. 10 no. 10 , pp. 3133-3138, Oct. 2011.

[6] H. Tran, H.-. Zepernick, and H. Phan, "Cognitive proactive and reactive DF relaying schemes under joint outage and peak transmit power constraints," IEEE Commun. Lett., vol. 17, no. 8, pp. 1548-1551, Aug. 2013.

[7] R. F. Schaefer and H. Boche, "Physical layer service integration in wireless networks: Signal processing challenges," IEEE Signal Process. Mag., vol. 31, no. 3, pp. 147-156, May 2014.

[8] M. Bloch, J. O. Barros, M. R. Rodrigues, and S. W. McLaughlin, "Wireless information-theoretic securiry," IEEE Trans. Inf. Theory, vol. 54, no. 6, pp. 2515-2534, Jun. 2008.

[9] Y.-S. Shiu, S. Y. Chang, H.-C. Wu, S. C.-H. Huang, and H.-H. Chen "Physical layer security in wireless networks: a tutorial," IEEE Wireless Commun., vol. 18, no. 2, pp. 66-74, Apr. 2011.

[10] L. Dong, Z. Han, A. P. Petropulu, and H. V. Poor, "Improving wireless physical layer security via cooperating relays," IEEE Trans. Signal Process., vol. 58, no. 3, pp. 1875-188, Mar. 2010.

[11] Y. Zou, X. Wang, and W. Shen, "Optimal relay selection for physical layer security in cooperative wireless networks," IEEE J. S. Areas Commun., vol. 31, no. 10, pp. 2099-2111, Oct. 2013.

[12] I. Krikidis, "Opportunistic relay selection for cooperative networks with secrecy constraints," IET Commun., vol. 4, no. 15, pp. 1787-1791, Oct. 2010.

[13] V. N. Q. Bao, N. Linh-Trung, and M. Debbah, "Relay selection schemes for dual-hop networks under security constraints with multiple eavesdroppers," IEEE Trans. Wireless Commun., vol. 12, no. 12, pp. 60766085, Dec. 2013.

[14] A. Attar, H. Tang, A. V. Vasilakos, F. R. Yu, and V. C. M. Leung, "A survey of security challenges in cognitive radio networks: solutions and future research directions," Proc. IEEE, vol. 100, no. 12, pp. 3172-3186, Dec. 2012.

[15] H. Sakran, M. Shokair, O. Nasr, S. El-Rabaie, and A. A. El-Azm, "Proposed relay selection scheme for physical layer security in cognitive radio networks," IET Commun., vol. 6, no. 16, pp. 2676-2687, Nov. 2012.

[16] I. Gradshteyn and I. Ryzhik, Table of Integrals, Series and Products, 7th ed. Elsevier, 2007 